\title{
Benzodiazepine Withdrawal - Does This Lead to an Increase in the Use of Antipsychotics?
}

\author{
Viggo Rask Kragh Jørgensen*
}

Lagehuset Thyborøn, Arøvej 1b, 7680 Thyborøn, Denmark

\begin{abstract}
Introduction: In 2004, two Danish GPs in the town of Thyborøn introduced a more restrictive approach to the prescription of benzodiazepines (BD) and cyclopyrrolones (CP). A prescription could only be renewed following personal consultation, and medication could only be prescribed for one month at a time. Every month, the practitioner and the patient had to consider whether current levels of consumption were appropriate or whether a reduction was to be implemented. This approach reduced the consumption of anxiolytics and hypnotics by $87 \%$ and $92 \%$, respectively, over a 3 year period. There is a general paucity of knowledge as to whether an intervention such as the one described above actually reduces drug consumption, or merely transfers consumption to other drugs, where especially antipsychotics (AP) are in the spotlight.
\end{abstract}

Materials and Methods: The current article describes the consumption of AP before and after the intervention. Consumption was followed via the Danish Medicines Agency's website Ordiprax, where one can determine the amount of prescription medications sold in pharmacies by individual medical practices.

Results: In both practices, a non-significant increase in the overall consumption of AP was observed during the course of the intervention against BD and CP. Although the consumption of some AP subgroups experienced a significant increase, no specific pattern could be observed.

Conclusion: The intervention against $\mathrm{BD}$ and $\mathrm{CP}$ did not result in a significant increase in total prescription volumes of AP. It cannot be excluded that the intervention influenced individual prescriptions.

Keywords: Benzodiazepines, Cyclopyrrolones, Antipsychotics, Sedative, Hypnotics, General practice.

\section{INTRODUCTION}

In recent years, the consumption of benzodiazepines (BD) and cyclopyrrolones (CP) has attracted increased attention. In 2003, a new national circular instructed Danish physicians to assess the prescription of hypnotics and anxiolytics after 1-2 and 3-4 weeks of consumption, respectively [1]. These rules were essentially in accordance with the instructions issued in England and Norway [2, 3].

Current rules for the prescription of hypnotics and anxiolytics are significantly stricter [4]. Where the Danish circular from 2003 exempted older patients, they have now become one of the main areas of focus for the reduction of the consumption of $\mathrm{BD}$ and $\mathrm{CP}$. Long-term treatment of the elderly with $\mathrm{BD}$ and $\mathrm{CP}$ may in principle no longer take place.

This attention is academically justified, as the consumption of these substances is associated with significant side effects [5]. The common denominators for many of these side effects are effects on cognitive functions, such as impaired memory and loss of concentration.

The hypnotic effect of $\mathrm{BD}$ and $\mathrm{CP}$ declines and disappears after a few months, while the anxiolytic effect almost disappears over the same period [6-8]. Thus, patients

*Address correspondence to this author at the Ærøvej 1b, 7680 Thyborøn, Denmark; Tel: 97 832493; (Office): 97 832300;

Fax: 97 832057; E-mail: VKJ@DADLNET.DK consume drugs that have no positive effect on long-term treatment, and the only long-term effects are significant associated complications. Addiction and the risk of withdrawal symptoms retain patients in their consumption patterns [9]. The most common withdrawal symptoms are restlessness, anxiety, insomnia, increased dream activity and depressed mood [10-12]. Severe withdrawal symptoms include depression, suicidal tendencies and withdrawal convulsions [5]. Almost all experience in this area has been obtained by specialist clinics, which treat the most affected patients [13]. This has led to a somewhat negative view of the use of this group of drugs.

The reduction in the prescription of $\mathrm{BD}$ and $\mathrm{CP}$ did not result in direct savings to the Danish National Health Insurance, as no subsidies were provided for these drugs. The price of these drugs is low by Danish standards, where the price of 100 Zopiclone tablets, for example, is 6,4 Euro. However, the indirect savings can be substantial. Studies suggest that each dependent costs society in the region of 48,000 Euro [12].

In 2003, practitioners at two medical practices in Thyborøn reacted to these problems related to the prescription of $\mathrm{BD}$ and $\mathrm{CP}$ [12]. In collaboration with Ringkøbing County Medicine Unit and the County Medical Health Officer, a few simple rules for the prescription of $\mathrm{BD}$ and $\mathrm{CP}$ were introduced in the two practices. These rules were essentially in accordance with the Danish National Board of Health's pre- 
vious and current recommendations [1, 4]. For the prescription of $\mathrm{BD}$ and $\mathrm{CP}$, the following recommendations were introduced:

- the discontinuation of telephone prescriptions

- prescription only following personal consultation

- prescription for a maximum of 1 month's consumption.

The introduction of these rules led to the patient and the practitioner re- evaluating on a monthly basis whether current prescription levels were appropriate and therefore should continue, or whether a withdrawal should be initiated.

In the 3-year period from 2004, the two practices were able to reduce the consumption of BD as anxiolytics by $87 \%$, and $\mathrm{BD}$ and $\mathrm{CP}$ as hypnotica by $92 \%$ [12]. Among colleagues, it was argued that consumption was merely transferred to antipsychotics (AP) instead. This argument is academically relevant, as evidence shows that other interventions have merely led to a shift toward the consumption of other drugs [14]. This shift may include antipsychotics and antidepressives, as well as changes in the consumption of alcohol, over-the-counter medications or illegal drugs.

The use of antipsychotic drugs in the treatment of anxiety disorders is generally considered to be poorly elucidated [15]. The Danish National Board of Health's reference program for anxiety disorders in adults recommends that patients suffering from anxiety disorders should be offered a proven, effective treatment in the form of antidepressants or cognitive behavioral therapy, or a combination of these treatments. The first choice among pharmacological treatments for all anxiety disorders apart from a few specific phobia is the use of newer antidepressants such as selective serotonin reuptake inhibitors (SSRI) [15].

Any shift in the consumption of BD and CP to antipsychotics may result in a significantly increased cost to the Danish National Health Insurance, since antipsychotics are generally subsidized by about $50 \%$. Treatment costs of antipsychotics vary; for example, the price of 100 DDD of Olanzapine is approximately 7 Euro while the price of 100 DDD of Ziprasidone is 152 Euro.

The use of antipsychotics may be linked to serious side effects, such as acute dystonia, Parkinsonism and tardive dyskinesia.

This article attempts to elucidate the question of whether the reduced consumption of $\mathrm{BD}$ and $\mathrm{CP}$ as described in the intervention above led to changes in the consumption of AP, through a systematic review of this group.

\section{MATERIALS AND METHODS}

Data were obtained from two practitioners in the Danish town of Thyborøn, comprising a total of 2275 patients at the start of the intervention. The data covers AP prescriptions for the years 2003-2006, where AP prescription data for 2003 is included to provide a baseline dataset.

The data compiled here were grouped according to their Anatomical Therapeutic Chemical (ATC) codes (Table 1). These groups facilitated a complete overview of antipsychotics registered in Denmark, and are included for the sake of completeness, without considering their direct relevance and usefulness as substitutes for BD and CP.
Table 1. The Classification of Antipsychotics According to their Anatomical Therapeutic Chemical (ATC) Classification System Codes.

\author{
$\underline{\text { 3. Level }}$ \\ N05A \\ Antipsychotic agents \\ $\underline{4 . \text { Level }}$ \\ N05AA \\ Phenothiazines with aliphatic side chains \\ Chlorpromazine \\ Levomepromazine \\ Acepromazine \\ N05AB \\ Phenothiazines with piperazine-structures \\ Fluphenazin \\ Perphenazin \\ Prochlorperazine
}

\section{N05AC}

Phenothiazines with piperidine structures

Periciazin

Thioridazine

N05AD

Butyrophenone derivatives

Haloperidol

Melperon

Pipamperon

N05AE

Indole derivatives

Sertindole

Ziprasidone

N05AF

Thioxanthen derivatives

Flupentixol

Chlorprothixene

Zuclopenthixol

N05AG

Diphenylbutylpiperidine derivatives

Pimozide

N05AH

Penfluridol

Diazepines and oxazepines

Clozapine

Olanzapine

Quetiapine

N05AK

Neuroleptics for tardive dyskinesia

Tetrabenozine

N05AL

Benzamides

Sulpiride

Amisulpiride

N05AN

Lithium

Lithium

N05AX

Other antipsychotics

Risperidone

Aripiprazole

The Internet site http://www.ordiprax.dk was used for the evaluation, since data were easily accessible and covered most of the required material. Some data was obtained di- 
rectly from the Danish Medicines Agency, as several of Ordiprax pages were over three years old and therefore not readily available.

The Ordiprax database comprises data reported by pharmacists to the Danish Medicines Agency Pharmaceutical Statistics Register for the sale of prescription drugs to individuals. For each prescription handled by the pharmacy, the prescribing physician's personal code, the patients' national registration number as well as a product-specific code containing information on the anatomical therapeutic chemical (ATC)-code, package size and total amount of Daily Defined Doses (DDD) in the package is registered.

Ordiprax is divided into two categories: county data and practice data. Practice data is the individual practice's prescriptions for their own patients, redeemed in Danish pharmacies, and can usually only be seen by the practitioner. County data is available to all and covers all prescription expeditions (including those from specialists and hospitals).

The annual average change in AP is calculated using quarterly data for the period from 2003 to 2006 . In order to facilitate comparison with data from the rest of the country, gender- and age-standardized data are chosen.

Practice data are compared using Analysis of Variance (ANOVA) followed by Student-Newmann-Keuls postANOVA test (SNK; $\mathrm{P}=0.05$ ).

In order to compare these data, corresponding values for the old Ringkøbing Amt are selected.

The comparison between practice and the county prescriptions is illustrated by bar graphs, where error bars indicate LSD (Least Significant Difference; $(\mathrm{P}=0.05)$ ) calculated using analysis of variance (ANOVA) followed by Student-Newmann-Keuls post-ANOVA test.

The interval from 2003 to 2006 is chosen as the two practices carried out major restructuring in 2007, where patient numbers increased by over 1000 patients. The new patients had not participated in the intervention to reduce the consumption of cyclopyrrolones and benzodiazepines, and data from this period are not directly comparable.

No special attention was directed to the prescription of antipsychotics during the intervention, neither from the reference group nor at the national level. None of the participating physicians received special training in the use of antipsychotics in connection with the stated period.

\section{RESULTS}

The total consumption of AP (N05A) did not increase significantly in any of the two practices (Table 2) during the course of the intervention. However, there was a numerical increase in the consumption of AP in practice 1 from 358 to 470 per year, corresponding to an average increase of $10.4 \%$ per year, while in practice 2 a somewhat smaller increase from 495 to 593 , corresponding to an average increase of $6.6 \%$ per year, can be observed. This is in contrast to the significant increase in the counties total consumption of AP (N05A) from 942 to 1048, corresponding to an average of $3.7 \%$ per year (Table 3 ). For practice 1 , the consumption of AP at the start of the intervention was $38 \%$ of county consumption levels, increasing to $45 \%$ after three years of the intervention. For practice 2, the figures were 53\% and 57\%, respectively. For both practices, the consumption of AP is thus considerably below the county average.

The primary drug prescribed in the N05AA substance group was Levomepromazine (Nozinan). Changes in the consumption of N05AA for practice 1 and 2 are numerically small, and the latter practice shows a small yet significant decrease (Table 2). At the county level a significant decrease in consumption of 7,9\% per year can be observed (Table 3). The primary drug prescribed in the N05AB substance group was Prochlorperazine (Stemetil). The decline in consumption in practice 1 is not significant, while ordinations ceased altogether for practice 2 . At the county level, a significant decrease of $10.2 \%$ per year could be observed. The primary drug prescribed in the N05AD substance group was Melperon (Buronil). The increase in consumption for this drug group is $142 \%$ for practice 1 , although this increase is not statistically significant. Ordinations for this group ceased altogether for practice 2. Prescription volumes were modest, however practice 1 was among the highest prescribers in the county in the fourth quarter of 2006. When asked directly, they stated that the substance was used for the treatment of older, restless patients. In practice 2 this group of drugs is no longer prescribed. At the county level, consumption decreased significantly by $6.5 \%$. For practice 1 , the average prescribed volume was no higher than the county average in 2006.

The primary drug prescribed in the N05AE substance group was Ziprasidone (Zeldox, Geodon). This group has only been prescribed since 2003, and only in practice 1 . When asked directly, this practice informed that prescription only takes place in connection with prescription renewals following instructions from a specialist in psychiatry, and is thus not a primary prescription. The numerical prescription volume corresponding roughly to the increase in the use of this group for practice 1 (Table 2) is represented by a single patient who is prescribed two DDD per day. In the drug group N05AF prescriptions were primarily for Chlorprothixene (Truxal) and Flupentixol (Fluanxol). Prescriptions for both practices can be seen to decline, where declines are greatest for practice 1. At the county level a slight, nonsignificant decline can be observed (Table 3).

Pimozide (Orap) was prescribed in drug group N05AG solely following instruction from a specialist in psychiatry. In practice 1 this drug was essentially absent (Table 2). In practice 2, a non-significant increase in consumption can be observed. At the county level, a non-significant decrease in consumption was recorded (Table 3). Prescriptions in the drug group N05AH covered clozapine (Clozaril, Clozapine, Leponex) and olanzapine (Zyprexa). This group was prescribed by both practices solely under the guidance of a specialist in psychiatry. Prescriptions for Practice 1 remained largely unchanged for the duration of the intervention, while practice 2 showed a non-significant decrease in prescriptions of $8.2 \%$ (Table 2). Table 3 reveals a significant increase in prescription volumes of $9.9 \%$ at the county level during the same period.

In the drug group N05AN prescriptions were for lithium (Litarex, Lithium Carbonate). These preparations were used by practice 2 exclusively and were prescribed by a specialist in psychiatry, although treatment was carried out under the supervision of the physician associated with practice 2 . At 
Table 2. Data from the two practices participating in the intervention for the prescribed volume of antipsychotics calculated as the average of the four annual quarters. The volumes indicated for each classification are gender and age standardized defined daily doses divided by year per 1000 individuals. For columns marked with an asterisk there is a significant $(\mathrm{P}=$ 0.05) change in prescription volume over the period 2003 to 2006, based on an Analysis of Variance (ANOVA) followed by Student-Newmann-Keuls (SNK) post-ANOVA test

Practice 1:

\begin{tabular}{|c|c|c|c|c|c|c|c|c|c|c|c|c|c|}
\hline & N05A & N05AA & N05AB & N05AC & N05AD & N05AE & N05AF & N05AG & N05AH & N05AK & N05AL & N05AN & N05AX \\
\hline 2005 & 317 & 15 & 21 & 0 & 2 & 149 & 115 & 7 & 3 & 0 & 0 & 0 & 6 \\
\hline 2006 & 470 & 23 & 20 & 0 & 25 & 147 & 98 & 15 & 132 & 0 & 0 & 0 & 11 \\
\hline
\end{tabular}

Practice 2:

\begin{tabular}{|c|c|c|c|c|c|c|c|c|c|c|c|c|c|}
\hline & N05A & N05AA & N05AB & N05AC & N05AD & N05AE & N05AF & N05AG & N05AH & N05AK & N05AL & N05AN & N05AX \\
\hline 2004 & 633 & 20 & 13 & 0 & 26 & 0 & 150 & 77 & 203 & 0 & 0 & 59 & 86 \\
\hline 2005 & 546 & 31 & 0 & 0 & 18 & 3 & 56 & 76 & 171 & 0 & 0 & 44 & 149 \\
\hline 2006 & 593 & 17 & 0 & 0 & 0 & 0 & 93 & 80 & 136 & 0 & 0 & 68 & 199 \\
\hline
\end{tabular}

Table 3. County data for the prescribed volume of antipsychotics calculated as the average of the four annual quarters. The volumes indicated for each classification are gender and age standardized defined daily doses divided by year per 1000 individuals. For columns marked with an asterisk there is a significant $(P=0.05)$ change in prescription volume over the period 2003 to 2006, based on an Analysis of Variance (ANOVA) followed by Student-Newmann-Keuls (SNK) post-ANOVA test

\begin{tabular}{|c|c|c|c|c|c|c|c|c|c|c|c|c|c|}
\hline & N05A & N05AA & N05AB & N05AC & N05AD & N05AE & N05AF & N05AG & N05AH & N05AK & N05AL & N05AN & N05AX \\
\hline 2005 & 1056 & 39 & 98 & 10 & 43 & 59 & 182 & 23 & 326 & 6 & 12 & 111 & 148 \\
\hline 2006 & 1048 & 34 & 84 & 5 & 39 & 55 & 155 & 22 & 362 & 6 & 12 & 112 & 163 \\
\hline
\end{tabular}

the county level, prescription volume levels remained constant throughout the period of the intervention (Table 3 ). Risperidone (Risperdal) was prescribed in drug group N05AX. In practice 1, consumption was highly variable, but declined by $25.7 \%$ over the course of the intervention (Table 2). In practice 2 , a significant increase in prescription numbers of $102.9 \%$ can be observed. Prescriptions in this group took place after consultation with a specialist in psychiatry. At the county level, prescriptions of this group increased by $34.9 \%$.

\section{DISCUSSION}

The overall increase in the prescription of AP reported for both practices is not statistically significant, although a numerical increase of $10.4 \%$ per year, corresponding to 112 DDD, can be observed for practice 1 . This increase can primarily be ascribed to prescription renewals of N05AE. How- ever, statistically significant prescription increases are apparent for the groups N05AA and N05AB, although the numerical increase for these groups in practice 1 are so small that from a practical point of view they are considered to be irrelevant for a proper assessment of the changes in these groups. In comparison, the total reduction in the prescription volumes of $\mathrm{BD}$ and $\mathrm{CP}$ over the same period was $4026 \mathrm{DDD}$ for practice 1 and 4438 DDD for practice 2, while the overall non-significant numeric increase in the consumption of AP for the two practices was $2.5 \%$ of the total reduction in the consumption $\mathrm{BD}$ and $\mathrm{CP}$ measured in DDD [12]. This should be viewed in light of the fact that county consumption levels of AP experienced a significant increase (Fig. 1). The increase of $6.6 \%$ in prescription volumes in the group N05AX for practice 2 is reflected in the county result. In contrast to these increases, several numerical declines in the prescription of AP are evident. For practice 1 it is reported 


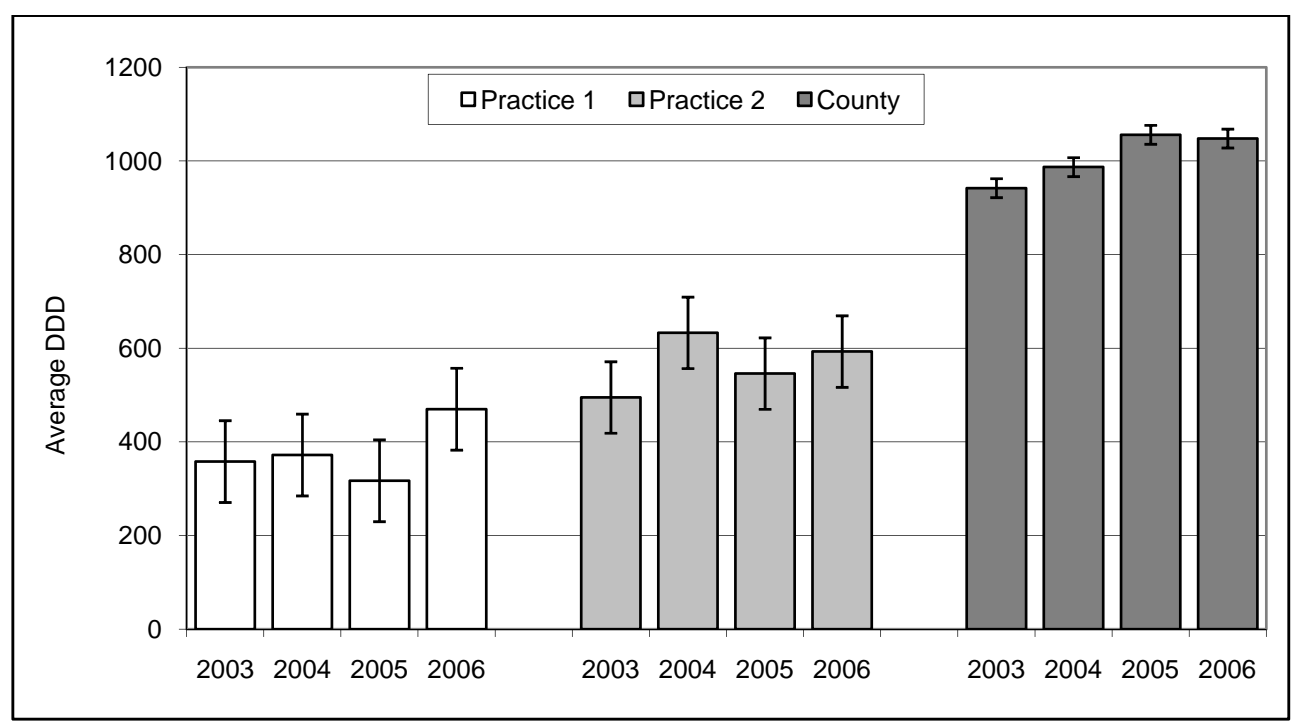

Fig. (1). The total prescription volumes of antipsychotics (N05A) by the two practices participating in the intervention, compared with county figures. The figures are quarterly averages for the given year given in defined daily doses per 1000 patients. Error bars indicate the LSD (Least Significant Difference; $(\mathrm{P}=.05)$ ) calculated using analysis of variance (ANOVA) followed by Student-Newmann-Keuls postANOVA test.

that the non-significant numerical increase in the prescription of N05AD may be due to a change in prescription practice from a BD to the N05AD Melperon (Buronil). Changes in the remaining groups are negligible.

In 1989, the State of New York conducted an intervention to reduce the consumption benzodiazepines [14]. The main components of this intervention were the introduction of regulations requiring that prescriptions of benzodiazepines be copied, as well as the implementation of stricter supervision of the prescription of this group of drugs. This led a 30$60 \%$ decrease in the consumption of $\mathrm{BD}$. The intervention was characterized by an element of coercion against physicians in the form of a requirement that prescriptions of $\mathrm{BD}$ be reported as well as copied in triplicate on pre-purchased forms. However, this lead to a shift toward the prescription of less appropriate medications. This prescription shift gave cause for introspection by the governing authorities.

The difference between the interventions in Thyborøn and in New York, in addition to differences in size, is that the intervention in Thyborøn was carried out on a voluntary basis [12], and that the physicians were motivated. The patient was at all times at the center of the intervention while prescription changes were carried out following a monthly consultation and were based on consensus. The negative aspects of the intervention in New York were not seen for the intervention in Thyborøn. We know from earlier studies that the intervention in Thyborøn had not lead to any increase in the use of antidepressants [16]. Any numerical increase in the use of AP during the intervention in Thyborøn was not statistically significant, and the numerical increases reported here can be attributed to the consumption of a few individuals.

In Thyborøn the consumption of antipsychotics was considerably below the county average. One explanation may be the lack of psychiatric institutions in the area and the migration of users of antipsychotics to areas that had these facilities. It should also be emphasized that this study does not take into consideration whether the level of treatment with antipsychotics in both practices is appropriate. This study focuses exclusively on the question of whether treatment with antipsychotics increased significantly due to the restrictive attitude toward the prescription of $\mathrm{BD}$ and $\mathrm{CP}$. The study does not provide evidence to explain why there was no concomitant increase in the consumption of antipsychotics. The simplest explanation could be that there was no need to use this drug group for this group of patients. This could be due to the fact that patients only needed the increased care and attention that came with the increased number of consultations.

A restrictive approach to the prescription of $\mathrm{BD}$ and $\mathrm{CP}$ does not necessarily mean that a practice's prescription of AP automatically increases uncontrollably, although it cannot be excluded that this may have an impact on individual prescriptions, however this study does not provide information regarding prescription changes for individual users or for changes in the number of users.

The Thyborøn-model was introduced with a minimum of extra work for the practitioners. In the beginning the two practices used 4-5 consultations per week per 1000 patients, and after three years of the intervention consultations fell to one consultation per week per 1000 registered patients. The added burden to the participating practitioners was minimal. No patients where hospitalized as a result of the intervention, and a single patient was referred to a practicing psychiatrist. No patients developed severe withdrawal symptoms such as suicidal impulses and withdrawal convulsions [12]. There were no official complaints to the Danish Public Health Service Complaints Board or to the Chief Medical Officer [10].

On the basis of the present as well as previous [16] studies, it can be concluded that there has been no significant increase in the prescription of anti-depressive or antipsychotic drugs as a result of the intervention. 
The intervention is cheap, easy to implement and leads to a minimum of medical discomfort for patients. A reduction in the prescription of $\mathrm{BD}$ and $\mathrm{CP}$ results in an improvement in quality of life while minimizing the psychological and physical damage caused by the intake of these drugs [9].

Finally, the findings of the intervention are reinforced by the fact that there were only four patients who changed physician because of the intervention.

\section{CONCLUSION}

The results presented here indicate that a reduction of $\mathrm{BD}$ and CP does not necessarily lead to a significant increase in the consumption of AP and support the assumption that a similar intervention may be successfully applied in other general practices

\section{ACKNOWLEDGEMENTS}

Thanks are extended to Dr. Hans Holmsgaard, Thyborøn for cooperation and for providing data and to Medicine Team, Central Denmark Region for financial support for the statistical analysis of the data as well as translation of this article.

\section{ETHICAL APPROVAL}

Not required, since the intervention was not a trial.

\section{REFERENCES}

[1] Communication regarding the prescription of addictive drugs. Directive CIR 12 from 13/01/2003. Copenhagen: Danish National Board of Health, 2003. [in Danish] [online]. Available from: https://www.retsinformation.dk/Forms/R0710.aspx?id=9483 [cited: $22^{\text {nd }}$ Oct 2011].

[2] Benzodiazepines warning, Chief Medical Officer's Update 37, Patient Safety, Department of Health, January 2004. Available from: http://www.benzo.org.uk/cmo.htm [cited: $22^{\text {nd }}$ Oct 2011].

[3] Addictive drugs. Prescription and justification [in Norwegian]. Norwegian Board of Health. Available from URL http://www.he- lsetilsynet.no/no/ts/sokeside/?quicksearchquery=14.9.2001 [cited $22^{\text {nd }}$ Oct 2011].

[4] Communication regarding the prescription of addictive drugs Danish National Board of Health, 2008. [in Danish][online]. Available from URL Available from: https://www.retsinformation.dk/Forms/R0710.aspx?id=117508 [cited: $22^{\text {nd }}$ Oct 2011].

[5] Ashton CH. Benzodiazepines: How they work and how to withdraw. The Ashton manual 2002. Available from: http://www.benzo.org.uk/manual/index.htm [cited: $22^{\text {nd }}$ Oct 2011].

[6] Gjerlach J, Vestergaard P. Psykofarmaka. 1. udgave. København: PsykiatriFonden 2006.

[7] Srisurapanont M, Critchley J, Garner P, Maneeton B, Wongpakaran $\mathrm{N}$. Interventions to reduce benzodiazepine prescribing. (Protocol) Cochrane Database of Systematic Reviews 2005, Issue 1. Art. No. CD005172. DOI: 10.1002/14651858.CD005172

[8] Systematic review of the benzodiazepines. Guidelines for data sheets on diazepam, chlordiazepoxide, medazepam, clorazepate, lorazepam, oxazepam, temazepam, triazolam, nitrazepam and flurazepam. Committee on the Review of Medicines. BMJ March 1980; 280: 910-12.

[9] Torper J, Steine S. Seponation of benzodiazepine- how is the experience of the users [In Norwegian] Tidsskr Nor Laegeforen 2004; 124(18): 2342-4.

[10] Jørgensen VRK, Toft BS, Fogh MS. Reducing the use of benzodiazepines and cyclopyrrolones in clinical practice! [in Danish] Ugeskr Laeger 2006; 168: 1636-40.

[11] Jørgensen VRK, Toft BS. Reduction in the use of benzodiazepines and cyclopyrrolones in general practice. Pharm Pract 2008; 6(3): $136-41$.

[12] Jørgensen VRK, Toft BS. Intervention against the excessive use of anxiolytica and hypnotica in two general practices. Open Drug Safety J 2010; 1: 1-5.

[13] Ashton H. Benzodiazepine withdrawal: outcome in 50 patients. $\mathrm{Br}$ J Addiction 1987; 82: 665-71.

[14] Weintraub M, Singh S, Byrne L, Maharaj K, Guttmacher L. Consequences of the 1989 New York State triplicate benzodiazepine prescription regulations. JAMA1991; 266(17): 2392-7.

[15] Reference program for anxiety disorders in adults. Copenhagen; Danish National Board of Health, 2007[In Danish]. Available from: http://www.sst.dk/publ/Publ2007/PLAN/SfR/SST_Angstrapport_w eb.pdf [cited: $22^{\text {nd }}$ Oct 2011].

[16] Jørgensen VRK. Benzodiazepine reduction does not lead to an increased consumption of antidepressants. A study from two medical practices. [In Danish] Ugeskr Laeger 2009; 171: 2999-3003. 